$40(1) \mid 2011$

Varia

\title{
Modernité, religion et identité dans le Nord Potosí
}

Le cas de l'ayllu Chiro

\section{Claude Le Gouill}

\section{OpenEdition}

Journals

Édition électronique

URL : http://journals.openedition.org/bifea/1687

DOI : 10.4000/bifea.1687

ISSN : 2076-5827

Éditeur

Institut Français d'Études Andines

\section{Édition imprimée}

Date de publication : 1 avril 2011

Pagination : 205-211

ISSN : 0303-7495

\section{Référence électronique}

Claude Le Gouill, « Modernité, religion et identité dans le Nord Potosí », Bulletin de I'Institut français

d'études andines [En ligne], 40 (1) | 2011, mis en ligne le 01 février 2012, consulté le 07 novembre 2020. URL : http://journals.openedition.org/bifea/1687 ; DOI : https://doi.org/10.4000/bifea.1687

Les contenus du Bulletin de l'Institut français d'études andines sont mis à disposition selon les termes de la licence Creative Commons Attribution - Pas d'Utilisation Commerciale - Pas de Modification 4.0 International. 


\section{Modernité, religion et identité dans le Nord Potosí Le cas de I'ayllu Chiro}

\section{Claude Le Gouill*}

La région andine bolivienne connaît un dualisme organisationnel provenant de I'opposition entre l'organisation pré-coloniale de l'ayllu1 et I'organisation syndicale paysanne. À la source de ce conflit sont souvent mis en avant les facteurs historiques de la colonisation espagnole avec, d'un côté, les « communautés libres » des terres d'altitudes (puna) ayant conservé leurs identités territoriales et, de l'autre, les haciendas des vallées où travaillait la main d'oeuvre indigène. De ces dernières émerge le syndicalisme paysan impulsé par la réforme agraire du Mouvement National Révolutionnaire (MNR) en 1953.

Un récent travail de terrain réalisé dans le Nord Potosí nous montre que ce dualisme repose également sur les conflits entre leaders pour le contrôle des campagnes. Dans I'ayllu Chiro, que nous étudions ici, il prend une tournure toute particulière par sa violence et par sa lutte autour du contrôle des « biens du Salut ».

\section{ASPECTS HISTORIQUES DE L'AYLLU CHIRO}

L'ayllu Chiro appartient à la municipalité de San Pedro de Buena Vista, un centre urbain situé dans les vallées de la province Charcas et contrôlé par les propriétaires des nombreuses haciendas de la région. Chiro formait sous l'époque coloniale le Jatun Ayllu Qhayana avec six autres ayllus, la majorité d'entre eux situés sur les terres d'altitude (fig. 1). Fidèle à la société segmentaire andine, le Jatun Ayllu

* Doctorant à I'Institut des Hautes Études de I'Amérique Latine (IHEAL), Paris 3, aide à la mobilité de I'Institut Français d'Études Andines (IFEA, UMIFRE 17, CNRS-MAEE) 2009-2011. E-mail: claudelegouill@yahoo.fr

1 L'ayllu est l’organisation territoriale et sociale andine possédant son propre système d'autorités. 


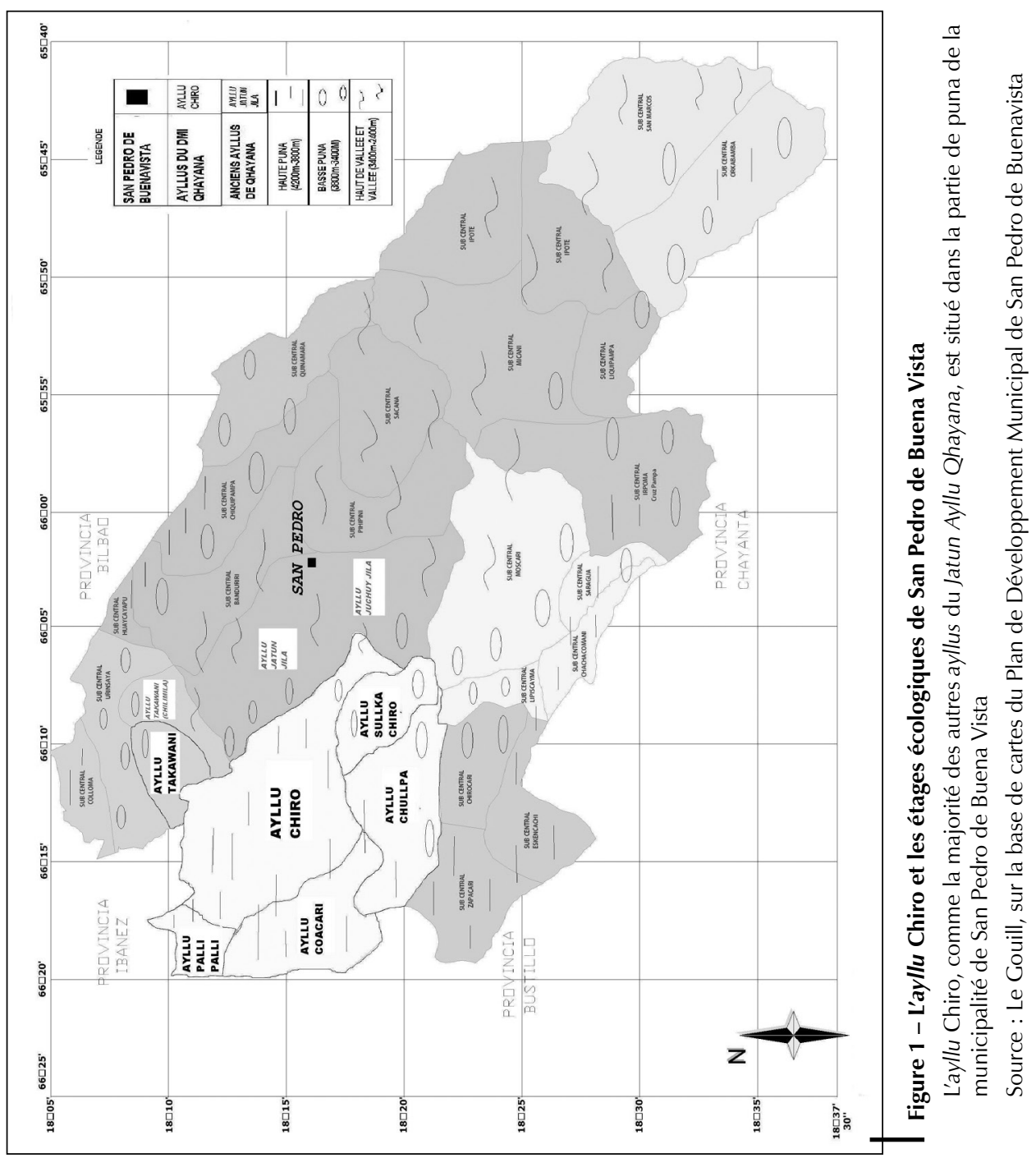


Qhayana appartenait à la marka Chayanta et à la nation Charka, une frange ethnique s'étalant des terres de puna de l'actuelle province Bustillo jusqu'aux vallées de San Pedro de Buena Vista.

Avec la réforme agraire, se développe le syndicalisme paysan de la Fédération Spéciale du Nord Potosí à partir de Banduriri, dans le canton de San Pedro de Buena Vista. Cette Fédération est née à l'initiative d'anciens mineurs de Llallagua et d'anciens travailleurs des haciendas (Harris \& Albó, 1976). Elle se fait cependant rapidement « récupérer » par les propriétaires terriens qui bloquent dès lors le processus de redistribution des terres des haciendas.

Il faut attendre la construction de la route Llallagua-Cochabamba pour que I'ayllu Chiro connaisse un changement significatif, avec la construction de son centre économique et politique, Chiro K'asa.

\section{CHIRO K'ASA : LA CONSTRUCTION RELIGIEUSE DU TERRITOIRE}

Dès sa naissance, le village de Chiro K'asa (fig. 2) marque un pas vers la modernité. Il naît dans les années 1960 avec la construction de la route et du campement ouvrier qui s'installe au lieu nommé Chiro K'asa. II n'existe à proximité que deux habitations où vivent Marcedonio Caysari et Inocencio Colque. Une troisième personne, Silvestre Sánchez, un propriétaire terrien fuyant l'agitation des vallées, y construit un petit commerce afin de vendre des produits de première nécessité aux ouvriers. De la volonté de ces «pionniers » va naître, peu à peu, un projet de centre urbain, économique et politique. À partir de la réorganisation territoriale autour de Chiro K'asa, se restructure la religion autour de la construction de nouveaux sites sacrés2 (église) et par l'institutionnalisation de la nouvelle fête patronale. Comme I'explique Mircéa Eliade, « l'installation dans un territoire équivaut à la fondation d'un monde » (Eliade, 1965 : 47). La place du village de Chiro K'asa est divisée en deux, avec d'un côté le cabildo de Chiro Grande et de l'autre Chiro Chico. C'est ainsi tout l'ayllu qui se réorganise autour de ce nouveau « centre du monde » où se créé des édifices publics (école, collège) et de nombreux commerces. La politique reste, quant à elle, contrôlée par les propriétaires terriens. Silvestre Sánchez, puis son beau-fils, se partagent la charge de corregidor durant une quinzaine d'année 3 et tentent de se démarquer du pouvoir de San Pedro de Buena Vista, non sans tension avec l'élite blanche-métisse des vallées, les vecinos.

\section{CRISE ÉCONOMIQUE ET ÉMERGENCE DE NOUVEAUX LEADERS}

En 1983 I'ayllu Chiro connaît une terrible sécheresse, comme toute la Bolivie. Silvia Rivera Cusicanqui a analysé cette crise (Rivera, 1992), montrant comment le

2 Sanchez voyage avec les élèves de l'école jusqu'au site du calvario (calvaire), un lieu sacré de l'ayllu Chiro, pour y chercher une pierre sacrée déposée ensuite dans l'église.

3 Le corregidor est une charge judiciaire traditionnellement occupée par l'élite métisse. 


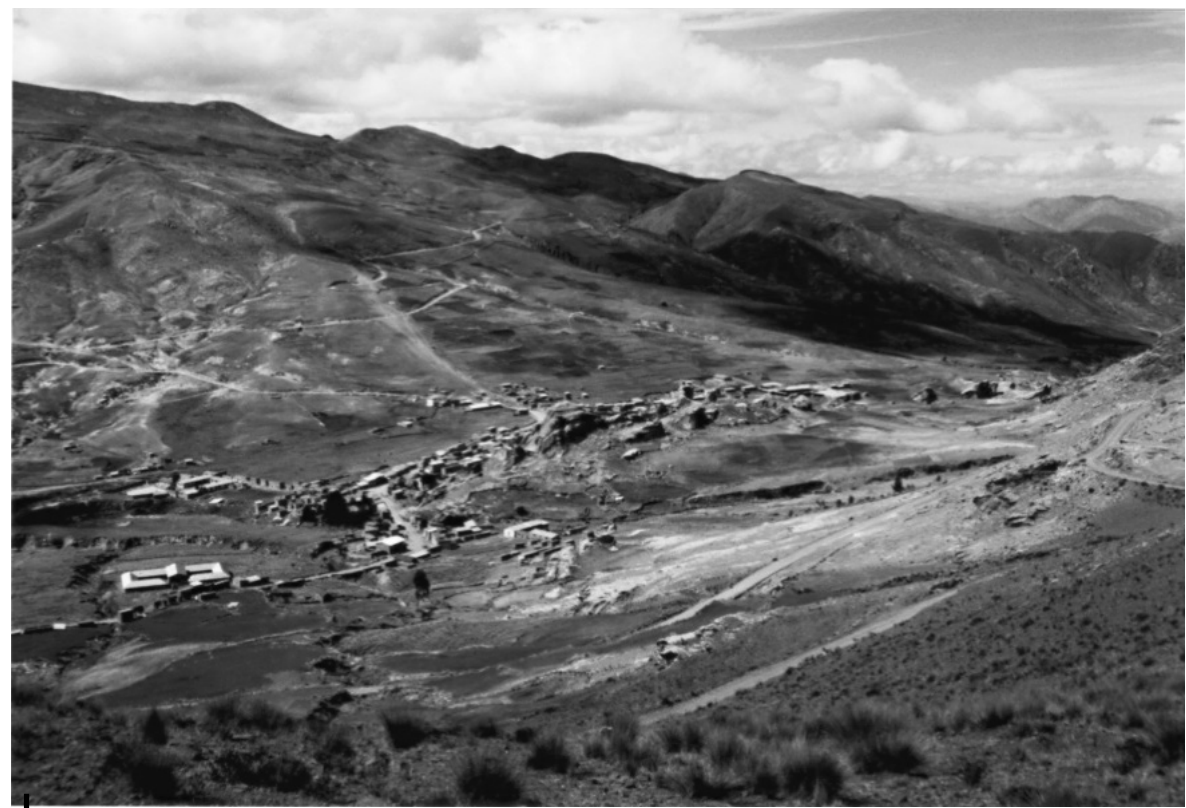

Figure 2 - Le village de Chiro K'asa

Situé à 3900 mètres d'altitude, Chiro K'asa s'est construit dans les années 1960 avec la construction de la route Llallagua-Cochabamba

Photo : Le Gouill

syndicalisme paysan a remplacé les ayllus de la province Bustillo par l'intermédiaire d'ONG et institutions soucieuses de « moderniser » les campagnes. Dans I'ayllu Chiro, I'ONG Vecinos Mundiales s'installe avec comme administrateur local Liborio Oporto, agronome de formation et fils de Sánchez.

Liborio Oporto impulse la construction d'un centre de formation de techniciens agronomes et de leaders syndicalistes rattachés à la Fédération Syndicale Unique des Travailleurs Paysans du Nord Potosi. Née en 1979, cette Fédération se détache de la Fédération Spéciale, liée aux gouvernements militaires par le «Pacte Militaire-Paysan », bien qu'elle garde certains liens avec les propriétaires terriens. Les anciens leaders de la Fédération Spéciale de Chiro sont rapidement écartés. Pour la première fois, la population locale s'accapare la charge de corregidor. Une nouvelle génération de leaders impulse des projets de développement dans l'ensemble des ayllus de puna. Chiro K'asa devient le centre du développement de la partie d'altitude.

Le projet des dirigeants syndicalistes poursuit aussi le chemin tracé par les « pionniers » de Chiro K'asa. En 1997, à la suite d'une longue lutte politique et juridique contre I'élite de San Pedro de Buena Vista, le Jatun Ayllu Qhayana reconstruit son territoire et son identité afin de devenir l'un des premiers District Municipal Indigène (DMI) de Bolivie. Chiro K'asa devient dès lors le centre régional du pouvoir. 


\section{DIEU SUR LE CHEMIN DE LA MODERNITÉ}

Si la route Llallagua-Cochabamba a entraîné un flux économique et idéologique vers Chiro K'asa, elle apporte aussi un nouveau courant religieux. Au début des années 1980, huit migrants reviennent à Chiro K'asa convertis à l'évangélisme. Ceux-ci parviennent peu à peu à convertir les « pionniers » de la construction du village et des familles ayant développé des entreprises (notamment dans le transport). Denise Arnold explique pour le cas des Qaqachakas que le phénomène évangéliste vient d'une volonté de chercher une nouvelle forme d'identification avec le commerce et de se délier de la vie paysanne (Arnold, 2008 : 114). Les premiers leaders évangélistes de Chiro proviennent d'une nouvelle élite économique, exclue du pouvoir politique avec l'apparition de la Fédération Unique, mais qui s'est forgée un capital économique par des pratiques extra-agricoles. L'évangélisme leur permet de reformuler leur identité et de retrouver un statut social au sein d'une communauté alternative. Le refus de consommer la chichat marque la principale frontière entre évangélistes et catholiques. L'économie faite sur la consommation de chicha doit être investie dans l'éducation des enfants.

Les conversions se propagent par la suite dans les communautés selon les liens de parentés de ce noyau central. Celles-ci interviennent peu après la sécheresse de 1983. Elles s'inscrivent dans une période de crise économique qui peut expliquer le refus de consommer la chicha et d'accomplir les obligations du cycle des fêtes catholiques. Les évangélistes continuent cependant de participer aux fêtes plus territoriales liées à l'ayllus. La construction et I'institutionnalisation du nouveau monde social repose à Chiro sur la socialisation primaire de la communauté agraire. II n'y a ainsi pas de véritable rupture avec l'organisation communautaire. Le nombre d'évangélistes augmente constamment jusqu'à la fin des années 1990 avec la peur de la « fin du monde » promis par plusieurs leaders de l'Église de Dieu Réforméé. A l'aube de l'an 2000, presque tout l'ayllu Chiro est converti. II ne reste qu'une poignée de catholiques, pour la plupart les dirigeants syndicaux liés à Vecinos Mundiales.

Une « fête spirituelle » est créée à Chiro K'asa et rassemble tous les ans les « frères » de l'Église de Dieu Reformée. La construction du temple évangéliste et la fête spirituelle marquent une tentative de se réapproprier le territoire religieux et de contrôler les «biens du Salut » (Arnold, 2008 : 147). Un missionnaire venu des États-Unis met en contact ce petit village avec le reste du monde durant cette fête. « Il vient des États-Unis ! » préviennent fièrement les fidèles.

La fin du monde n'étant jamais arrivée, beaucoup retournent au catholicisme dans les années 2000. L'ayllu dans son ensemble ne serait aujourd'hui qu'à $50 \%$

4 Alcool de maïs traditionnellement consommé lors des fêtes communautaires.

5 C'est le cas notamment de leur participation au cabildo, la fête durant laquelle les familles payent la contribution indigène leur donnant le droit à l'usage de la terre.

6 L'Église de Dieu Réformée évoque en effet la « fin du monde», sans pour autant donner de dates, contrairement aux leaders de Chiro qui ont profité de l'arrivée de l'an 2000 pour « recruter » de nombreux sympatisants. 
évangéliste, ce qui reste supérieur aux estimations nationales pour l'ensemble de la Bolivie7. Malgré cette diminution, Chiro K'asa est devenu le centre religieux de l'Église de Dieu Réformée pour tout le département de Potosí. D'après les fidèles, cette Église compterait aujourd'hui 30 temples dans le Nord Potosi pour un total de 1500 croyants.

\section{LES PARADOXES DE LA MODERNITÉ}

La lutte pour le contrôle des « biens du Salut » et du développement passe par I'accès aux charges politiques et par l'organisation communautaire. Écartés du pouvoir lors des élections municipales de 1999 par les leaders syndicalistes (catholiques) liés à Vecinos Mundiales, l'élite évangéliste décide de former sa propre organisation. Elle se rattache à la Fédération des Ayllus, Originaires et Indigènes du Nord Potosí (FAOINP), née en 1993. Celle-ci prône la « reconstitution des ayllus » et valorise la « cosmovision andine », ce qui n'est pas sans contradiction avec le mouvement évangéliste qui refuse la consommation de chicha et de feuille de coca. Le « retour à l'ayllu » repose plus sur un conflit entre des leaders évangélistes ayant une formation académique et des leaders syndicalistes liés au monde des ONG. Se construit cependant un discours dans lequel l'évangélisme est un acte de résistance contre la domination de l'Église catholique contrôlée par les propriétaires terriens, tout comme le « retour à l'ayllu » est une rupture avec le contrôle des vecinos sur le syndicat paysan. La conversion à l'évangélisme marque aussi une tentative de retour à une origine harmonieuse que le progrès technique a violé jusqu'à menacer aujourd'hui l'existence humaine. Ce retour à I'harmonie n'est ainsi pas si éloigné du pachakutis prôné par certains indianistes. Enfin, l'évangélisme et le retour à l'ayllu s'inscrivent dans un processus de modernisation des campagnes, intégrées à la fois au missionnaire américain et aux lois internationales sur les peuples indigènes.

À partir de l'année 2005, I'ayllu Chiro connaît un violent conflit entre les syndicalistes catholiques et les aylluistes évangéliques. Ce conflit se propage aux autres ayllus de Qhayana. Le thème religieux en est cependant exempt. Dans les autres ayllus, la reconstitution de l'ayllu repose en effet plus sur des conflits entre leaders et sur la lutte contre les propriétaires terriens. En 2007, les deux groupes s'affrontent à la dynamite lors d'un rassemblement syndical à Chiro K'asa. Lors du carnaval de Chiro K'asa, fête catholique impulsée par le syndicat, les évangélistes menacent de crucifier certains leaders syndicalistes9. Suite à cela, les deux organisations

7 Le recensement de 2001 montre que 77,8 \% de la population déclare appartenir à l'Église catholique, 16,5 \% à une église protestante et 3,6 \% à d'autres églises chrétiennes.

8 Nous pouvons décrire brièvement le pachakuti comme le retour à l'harmonie entre l'homme et la nature et par celui du pouvoir indigène.

9 Les évangélistes refusent de voir dans la croix catholique un symbole sacré, seule la communication directe avec dieu relève selon eux du sacré. La crucifixion serait ainsi une manière de combattre le mal par le mal. 


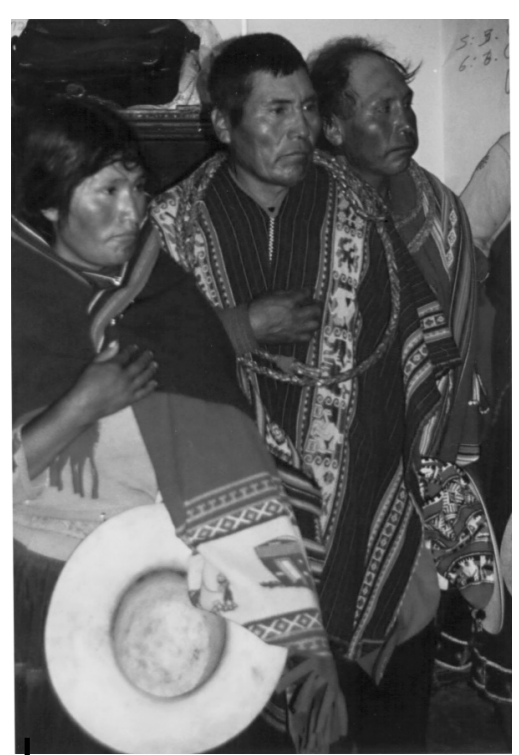

Figure 3 - Changement des autorités originaires de l'ayllu Chiro

Avant de prendre position, celles-ci doivent prêter serment, la main sur le coeur, le bras levé ou encore en faisant le signe de la croix, selon leur croyance religieuse ou politique

Photo : Le Gouill

entrent dans une lente pacification entraînant, du moins officiellement, la fin de l'organisation syndicale. Le DMI Qhayana est aujourd'hui contrôlé en quasi totalité par les aylluistes. Plusieurs leaders syndicaux intègrent l'organisation de l'ayllu mais restent cependant toujours exclus de la charge maximale de Segunda Mayor (fig. 3). Qhayana montre ainsi la force de la reconstruction de l'identité territoriale indigène. Après avoir été l'un des tous premiers DMI de Bolivie, Qhayana est parvenu lors des élections municipales de 2010 à conquérir pour la première fois le pouvoir à San Pedro de Buena Vista.

\section{CONCLUSION}

Le récent travail de terrain réalisé à Chiro K'asa montre la complexité des campagnes boliviennes. Il ne s'agit pas d'un monde rural intact face à la modernité, mais au contraire d'un monde cherchant à s'appuyer sur la base communale pour impulser le changement social. C'est dans ce sens que le processus de « reconstitution des ayllus » rencontre toujours plus d'adeptes. Ceux-ci ne sont pas tous évangélistes, bien au contraire. La violence du conflit qui secoua Chiro peut s'expliquer cependant par cette accumulation de tensions.

L'arrivée massive des ONG et l'accès à l'éducation ont bouleversé les rapports de domination ville/campagne pour laisser place à un conflit rural/rural au sein de la nouvelle élite paysanne-indigène. La religion et l'ethnicité sont de nouveaux instruments dans cette lutte pour le contrôle des campagnes, en appuyant leur idéologie autour des thèmes de la modernité et du changement social. L'analyse de I'ayllu Chiro nous montre ainsi tout autant les tensions internes aux communautés que leur force de mobilisation politique.

\section{Références citées}

ARNOLD, Y. D., 2008 - Entre los muertos, los diablos y el desarrollo en los Andes, 198 p.; La Paz : Instituto Superior Ecuménico Andino de Teología (ISEAT).

ELIADE, M., 1965 - Le sacré et le profane, 185 p.; Paris : Gallimard.

HARRIS, O. \& ALBÓ, J., 1976 - Montejas y guardatojos: Campesinos y mineros en el norte de Potosí, 89 p.; La Paz : Centro de Investigación y Promoción del Campesinado (CIPCA). Cuadernos de Investigación $n^{\circ} 7$.

RIVERA CUSICANQUI, S. \& Taller de Historia Oral Andina (THOA), 1992 - Ayllus y proyectos de desarrollo en el Norte de Potosí, 195 p.; La Paz : Aruwiyiri. 